\title{
Automatic Services Composition in the Grid Environments ${ }^{\star}$
}

\author{
Wenju Zhang, Fei Liu, Shudong Chen, and Fanyuan Ma \\ Shanghai Jiaotong University, Shanghai, \\ P.R. China, 200030 \\ \{zwj03, liufei001, chenshudong, fyma\}@sjtu.edu.cn
}

\begin{abstract}
Different planning techniques have been proposed so far which address the problem of automated composition of web services. However, in realistic cases, the planning problem is far from trivial. Such issue is more serious when services are embraced in grid environments, which are usually resource-conscious. We propose a planning techniques for the automated composition of grid services described in OWL-S process models. The technique allows for the synthesis of plans that encode compositions of grid services with the usual programming constructs. We apply this technique in our DDGrid project and do some preliminary experimental evaluations.
\end{abstract}

\section{Introduction}

Currently, the problem of the composition of services is addressed by two orthogonal efforts from the industry and the Semantic Web community. Our research aims to develop intelligent middleware components that encapsulate the expertise required to use grids. Earlier, we used AI planning techniques to automatically generate executable job workflows from high-level specifications of desired results. We integrated our planner in a grid environment to extract relevant knowledge from existing grid middleware.

The rest of this paper is organized as follows. Section 2 introduces the grid environments of our techniques apply. Section 3 presents how OWL-S process models can be translated into state transition systems and the principle and definition of automatic grid services composition. Section 4 provides an example and a preliminary experimental evaluation. Section 5 discusses the relevant ontologies and planning techniques. We conclude in Section 6.

\section{Grid Environments and Integration}

Middleware services such as those that the Globus toolkit provides help users obtain information about available resources, component software, data files and

\footnotetext{
* This research work was supported in part by the the National High Technology Research and Development Program of China (863 Program), under Grant No. 2004AA104270.
} 
the execution environment. We have used several of these services as knowledge sources for our planner as part of its integration in the DDGrid (Drug Discovery Grid, www.ddgrid.ac.cn) project for planning and execution in grids. An AI planner and the typical services composition environment shown in Figure 1.

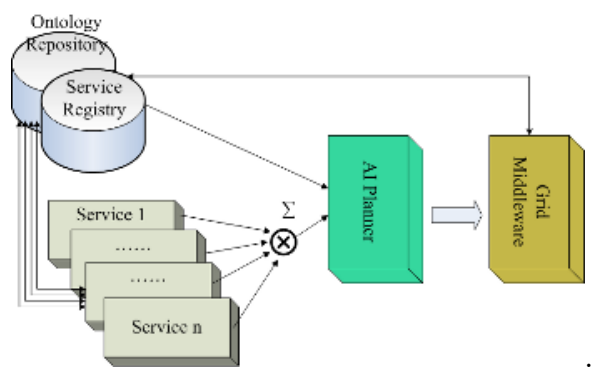

Fig. 1. An AI planner and the typical services composition environment

A complete planning-based solution for Grid or Web services composition must address execution as well as construction of workflows. Grid and Web planning systems make decisions in dynamic environments in which the services that are composed as part of a plan may become unavailable during its execution.

Finding an optimal allocation of processors for tasks in a workflow is NP-hard and tools must focus on finding reasonable heuristics or on identifying families of problems that can be solved efficiently. In a dynamic environment, the system needs to make time-dependent decisions when an initial workflow allocation is found to be inappropriate during execution.

\section{OWL-S Process Models and Automated Composition}

OWL-S process models are declarative descriptions of the properties of web service programs. Process models distinguish between atomic processed and composite processes as mentioned before.

We encode OWL-S process models as state transition systems, which describe dynamic systems that can be in one of their possible states and can evolve to new states as a result of performing some actions.

The execution structure corresponding to domain $\Sigma$ and plan $\pi$ is the Kripke structure $\Sigma_{\pi}=\left\langle S, S_{0}, R\right\rangle$, where:

$-S$ is the set of configurations;

$-S_{0} \subseteq P$ are the initial configurations;

$-R \subseteq S \times S$ are the transitions between configurations.

The execution structure $\Sigma_{\pi}$ represents the evolutions of the domain $\Sigma$ controlled by the plan $\pi$. It is the execution structure $\Sigma_{\pi}$ that must satisfy the composition goal $G$. If $\Sigma_{\pi} \models G$, we say that $\pi$ is a valid plan for $G$ on $\Sigma$. 
In presence of partial observability, at each plan execution step, the plan executor has to consider a set of domain states, each equally plausible given the initial knowledge and the observed behavior of the domain so far. Thus in general, given a belief $B$, performing an action $a$ and taking into account the obtained observation $o$ gets to a new belief $\operatorname{Evolve}(B, a, o)$ :

$$
\operatorname{Evolve}(B, a, o)=\left\{s^{\prime}: \exists s \in B . s^{\prime} \in \mathcal{T}(s, a) \wedge \mathcal{M}\left(s^{\prime}\right)=o\right\}
$$

Planning in this framework consists in searching through the possible evolutions of initial beliefs, to retrieve a conditional course of actions that leads to beliefs that satisfy the goal.

\section{An Example and Experimental Evaluation}

Our techniques are general, but we have applied them in the context of DDGrid project. The Drug Discovery Service (as shown in Figure 2), also called Composed Grid Service (CGS), is composed by three related grid services we provided: a Virtual Chemical DataBase Data Service, a Dock Service and a Payment Service. The idea is that of combining these three services so that user may directly request the CGS to complete the molecule docking task.

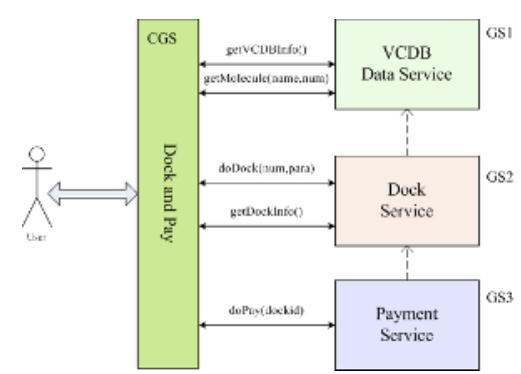

Fig. 2. A DDGrid Services Composition Example

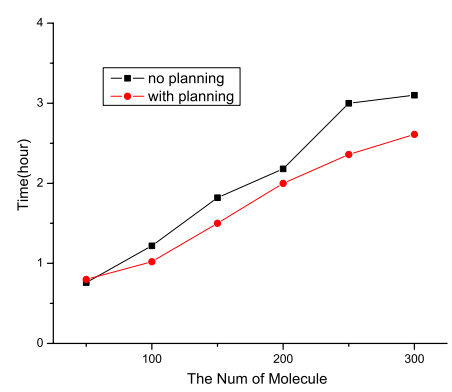

Fig. 3. The Execution Time of the CGS with Different Number of Molecule

The Initial State: The planner receives as input an initial state that captures information from several sources, including

- Hardware/Software resources available to the user. those information obtain from the grid middleware of DDGrid.

- The dock application's specifical parameters. such as the protein file description, the ligand atom file description, etc.

- Estimates of computation power of every Virtual Organization and bandwidths between the resources.

- The identification of the grid user and the authorization policy. 
Composition Goals: The composition goals express requirements for the services to be automatically generated. In our example (see Figure 2), The CGS should try to reach the ideal situation when the user has submitted his job, and the VCDB data service has the corresponding molecule to dock. Finally, the dock grid service finish the job and the user pay for it through the payment service.

We have executed the composed grid service in our DDGrid platform. The planner performed 6 actions, docking 1050 molecules. The effectiveness and performance can be seen partially from Figure 3 .

\section{Related Work}

Several projects have recently demonstrated planning techniques for Web and Grid Services. Other projects use knowledge bases to facilitate Grid use. The myGrid project uses OWL and OWL-S to describe application components as Semantic Web Services. These descriptions support matching and discovery of components through a description logic reasoner.

Other planning techniques have been applied to related problems in the filed of grid and web services [1, 3, 4]. The interactive composition of information gathering services has been tackled in [2] by using Constraint Satisfaction Problems techniques.

\section{Conclusion and Future Work}

In this paper, we have shown how ontologies and OWL-S process models can be used to generate automatically new composed grid services. This is achieved by translating OWL-S process models to state transition systems and by generating automatically a plan that can express conditional and iterative behaviors of the composition. Our preliminary experimental evaluation shows the potentialities of the approach.

\section{References}

1. J. Blythe, E. Deelman, and Y. Gil, Automatically Composed workflows for Grid Environments, IEEE Intelligent Systems, 2004.

2. S. Thakkar, C. Knoblock, and J.L. Ambite. A View Integration Approach to Dynamic Composition of Web Services, In Proceedings of ICAPS'03 Workshop on Planning for Web Services, 2003.

3. William K. Cheung et al., Towards Autonomous Service Composition in A Grid Environment, In Proceedings of the IEEE International Conference on Web Services(ICWS'04), 2004.

4. Tatiana Kechkaylo, Planning for Grid Applications with Explicit Reservations, The 14Th International Conference on Automated Planning \& Scheduling(ICAPS'04), 2004. 